Service social

\title{
The Lost and the Lonely - Homeless Women in Montreal, par Aileen D. Ross, Montréal, The Canadian Human Rights Foundation, 1982, 121 pages.
}

\section{Marc-André Delisle}

Volume 34, numéro 1, 1985

Personnes âgées, milieux de vie et pratiques sociales

URI : https://id.erudit.org/iderudit/706263ar

DOI : https://doi.org/10.7202/706263ar

Aller au sommaire du numéro

Éditeur(s)

École de service social de l'Université Laval

ISSN

1708-1734 (numérique)

Découvrir la revue

Citer ce compte rendu

Delisle, M.-A. (1985). Compte rendu de [The Lost and the Lonely - Homeless

Women in Montreal, par Aileen D. Ross, Montréal, The Canadian Human Rights Foundation, 1982, 121 pages.] Service social, 34(1), 190-191.

https://doi.org/10.7202/706263ar d'utilisation que vous pouvez consulter en ligne.

https://apropos.erudit.org/fr/usagers/politique-dutilisation/ 
The Lost and the Lonely. Homeless Women in Montreal, par Aileen D. Ross, Montréal, The Canadian Human Rights Foundation, 1982, 121 pages.

L'univers des personnes qui n'ont pas de foyer (homeless), et en particulier celui des femmes qui sont dans cette situation, est encore assez mal connu parce que trop peu de chercheurs l'ont exploré. Quelques travaux ont été effectués sur le sujet aux États-Unis, notamment ceux de H.M. Bahr, T. Caplow et S.E. Wallace. À ce que nous sachions, il n'existe pas d'études canadiennes comparables. Aussi l'ouvrage d'Aileen Ross vient-il combler un grand vide dans la sociographie d'ici.

Elle s'est intéressée à deux refuges pour femmes sans foyer situés à Montréal. Le premier, "Chez Doris" - du nom d'une prostituée assassinée en 1974 - n'est ouvert que durant la journée, alors que le second, situé dans la maison mère des Soeurs Grises, l'est continuellement. Madame Ross a procédé par observation participante, c'est-à-dire qu'elle a recueilli ses informations à travers des conversations avec les femmes sans foyer, le personnel des refuges et les professionnels qui gravitent autour de ces derniers. Il n'y a pas eu d'échantillonnage et aucun questionnaire n'a été administré. De toute façon, les méthodes quantitatives auraient été inappropriées puisque la population de référence est très mouvante. Le contexte créé par un tel type d'étude aurait également pu modifier, chez les femmes concernées, l'attitude envers les refuges. En procédant ainsi, il devenait toutefois impossible de vérifier des hypothèses. Cet ouvrage est donc principalement descriptif et comprend sept chapitres, qui sont autant de facettes différentes du problème de l'errance des femmes.

L'auteure commence par définir les concepts fondamentaux de sa recherche, en évoquant la littérature étrangère pertinente, puis décrit les deux refuges et leur environnement. Elle énumère ensuite les caractéristiques de certaines femmes qui les fréquentaient en 1977-1978: la moyenne d'âge est de 38 ans; à peine $12 \%$ ont moins de 20 ans et $11 \%$ plus de 60 ans. Le quart de ces femmes ont une santé mentale déficiente et il y en a, parmi elles, qui ont reçu des traitements psychiatriques; elles sont souvent anxieuses, déprimées, passives, confuses ou, au contraire, agressives et violentes et ont même parfois des tendances suicidaires ou, dans des moments de colère, peuvent-elles aller jusqu'à tuer (pp. 60-61).

Autre problème majeur auquel ces femmes sont confrontées: l'alcoolisme. Se joindre à un groupe qui boit est d'ailleurs, pour elles, un moyen d'échapper à la solitude. Elles ont peu de relations avec les membres de leur famille ou ces relations sont de mauvaise qualité ; la plupart du temps elles ont été rejetées par leur entourage. Incidemment, le rejet dont elles étaient victimes les enfermait dans le cercle vicieux de leur marginalité. Leur isolement les perturbait affectivement et les amenait à se comporter de manière asociale, ce qui les coupait du reste de la société. De plus, les femmes en cause ne parvenaient pas à se loger adéquatement; nombre d'entre elles passaient la nuit à la belle étoile, dans des endroits publics (quand elles y étaient tolérées), dans des refuges, en 
prison ou encore, si elles étaient assez jeunes et attirantes, chez un ou plusieurs hommes. Celles qui avaient un petit peu d'argent vivaient en chambre dans des sous-sols mal chauffés et pleins de vermine. Une certaine proportion de femmes sans foyer se droguaient, soit avec des médicaments, soit avec des narcotiques, et elles se liaient souvent à des trafiquants. Les refuges recevaient des ex-détenues dont la présence était particulièrement pénible parce que leur dossier judiciaire les stigmatisait.

La sociologue a consacré un chapitre à la manière dont les femmes sans foyer s'y prennent pour survivre. Cela va de la prostitution aux ruses et tromperies de toutes sortes, en passant par le vol et la fraude. La lecture de ces pages donne l'impression que le milieu décrit est peuplé d'individus qui subsistent en s'exploitant mutuellement. Qu'il y ait peu de relations et beaucoup d'incompréhension entre les clientes et les responsables des ressources communautaires devient alors aisément compréhensible. Les premières ne partagent les valeurs des secondes et ne parlent pas leur langage. Les femmes sans foyer ignorent leurs droits et ne savent généralement pas comment composer avec la bureaucratie. En conséquence, les services qu'elles reçoivent sont loin d'être proportionnels à leurs besoins. Il faudrait que plusieurs d'entre elles soient hébergées, en permanence, dans des hôpitaux généraux, des institutions psychiatriques ou des refuges pour miséreux; or les places sont rares dans ces établissements spécialisés et quantité de femmes sans foyer sont incapables de fonctionner dans un tel cadre ; c'est pourquoi elles sont souvent condamnées à vivre dans la rue jusqu'à ce que la mort les emporte. Leur réhabilitation est très difficile, et le travail auprès d'elles est peu motivant et peu valorisant pour les professionnels parce que les résultats tangibles tardent à venir et que la société n'est pas convaincue de l'utilité de cette tâche. Aux yeux du commun des mortels, ces sans-abri ne sont-elles pas irrécupérables? N'apparaissent-elles pas comme des êtres incapables de vivre heureuses, qui ne pourront jamais rien donner à la collectivité ? S'en occuper n'est-il pas alors un pur gaspillage d'énergie? La réponse à ces interrogations réside, en grande partie, dans les questions elles-mêmes. En effet, l'intérêt que suscite une étude comme celle de Madame Ross est à la fois significatif et rassurant car il permet de constater que le sort des citoyens de notre "Éthiopie intérieure" ne laisse pas tout le monde indifférent.

Université du Québec à Chicoutimi.

Marc-André DeLISLE

Femmes et politique, par Yolande CoHen (éd.), Montréal, Jour, 1981, 227 pages. ("Idéelles».)

Juin 1980, Université du Québec à Montréal, congrès des Sociétés savantes : des femmes de toutes disciplines se réunissent et se donnent une journée d'étude et de réflexion sur les pratiques culturelles, sociales et politiques des femmes d'aujourd'hui. Yolande Cohen a réuni les textes des principales 